\title{
Nonlinear Channels Characterization in OFDM Systems using Spectral Notch Signals
}

\author{
C. Venkata Sudhakar ${ }^{1}$, K.B. Jaya Prakash ${ }^{2}$ \\ ${ }^{I}$ Department of Electronics and Communication Engineering, \\ Sree Vidyanikethan Engineering College, TIRUPATI - 517 102, A. P., INDIA \\ ${ }^{2}$ Department of Electronics and Communication Engineering, \\ Sree Vidyanikethan Engineering College, TIRUPATI - 517 102, A. P., INDIA
}

\begin{abstract}
In this paper, we consider the problem of characterizing nonlinear channels in real baseband OFDM communication systems. A novel method is proposed to estimate the frequency domain Volterra kernels of nonlinear channels using OFDM signals with spectral notches. In this method, the OFDM signal is arranged in a way that each full-spectral OFDM symbol frame is followed by copies of the same OFDM symbol frame but each has a spectral notch at a distinct frequency. A mathematical expression of the difference signal between the outputs of a full-spectral OFDM symbol frame and its corresponding OFDM symbol frame with a spectral notch is derived. We find that, through the use of OFDM symbol frames with spectral notches, the task of Volterra kernel estimation can be decomposed into independent subtasks with smaller numbers of kernel coefficients. Therefore, the estimation of the Volterra kernels in the subtasks can be accomplished with more accuracy and less computational complexity using the same amount of available input and output data. We show that, the proposed method has a computational complexity of $\left(M^{2}\right)$, while the conventional method would require a computational complexity of $O\left(M^{4}\right)$.This indicates that a great saving in computation can be achieved by the proposed method without sacrificing the performance. The outperformance of the proposed method over the conventional method is justified by computer simulations. Real baseband OFDM systems, such as powerline and ADSL communications, can benefit from the proposed method.
\end{abstract}

\section{Introduction}

Orthogonal frequency-division multiplexing(OFDM) [1] is a well-developed modulation scheme for wideband communication applications. It has been adopted by many wireline and wireless communication standards to achieve high-speed data transmissions. Examples of such include HomePlug for powerline communications (PLCs) [2], ITU G.992.1 for asymmetric digital subscriber line (ADSL) communications [3], IEEE 802.11a/g for wireless LANs [4], DAB for digital audio broadcasting [5], and DVB for digital video broadcasting [6]. A unique feature of the OFDM signal is its high peak-to-average power ratio (PAR) caused by possible constructive combination of the subcarriers. The high PAR makes the OFDM signal very sensitive to nonlinearities of power amplifiers in a communication link. As a result, the output OFDM signal from a power amplifier can be corrupted by in-band distortions and out-of-band radiations. This poses many problems in the design of the OFDM receiver. Therefore, characterizing the nonlinear communication channel to understand how the channel nonlinearities distort the OFDM signal is essential to the success of the OFDM system.

One commonly used model for characterizing nonlinear channels is the Volterra series [7], [8]. Modeling a nonlinear channel with a Volterra series involves identifying the time-domain or the frequencydomain Volterra kernels of the channel. Note that the number of the required kernel coefficients is often very large even for a relatively low-order Volterra model. This makes the estimation of the Volterra kernel coefficients a challenging task. Early works on identifying Volterra kernels of nonlinear systems were based on the assumption that the input signal to the nonlinear system was Gaussian [9]-[11]. Although the Gaussianity assumption on the input signal greatly simplifies the involved mathematics for estimating the Volterra kernels, whether a sufficiently Gaussian input signal can be generated for obtaining a satisfactory result is often in doubt. A more general approach which estimated the Volterra kernels by solving a minimum mean square error (MMSE) problem was later developed [12]-[14]. Although this approach can use a broader class of random signals as the input, it requires estimation of various higher-order auto-moments (or auto-moment spectra) [15], [16] of the input and calculation of an inverse matrix. This leads to great complexity in terms of implementation and computation, especially when the number of Volterra kernel coefficients is large.

For identifying Volterra kernels of nonlinear channels in OFDM systems, methods based on the assumption that the OFDM signal is asymptotically i.i.d. complex Gaussian can be found in the literature [17], [18]. These methods were derived based on properties of higher-order cumulants of the complex Gaussian signal. In practice, the cumulants are estimated by taking time averages over a realization of the input and output data. Therefore, the estimated higher-order cumulants of the OFDM signal can only satisfy the higher- order 
cumulant properties of the complex Gaussian signal when the amount of data is sufficiently large. This suggests that a large amount of data is often required by these methods to obtain a satisfactory result. The demand for data can soon become intimidating when the number of involved Volterra kernel coefficients increases.

In this paper, we propose to estimate the frequency-domain Volterra kernels of nonlinear channels in real baseband OFDM systems by deploying spectral notches in the OFDM signal. Signals containing spectral notches are commonly used in multitone power ratio (MTPR) tests for determining the signal-to-distortion ratio of a discrete multitone system [3]. By using the difference signal yielded from the channel outputs of two OFDM signals, one with a spectral notch and the other without, we show that a frequency-domain Volterra kernel estimation task involving a large number of kernel coefficients can be decomposed into independent kernel estimation subtasks, each involving a significantly smaller number of kernel coefficients. Due to the small number of kernel coefficients, each individual kernel estimation subtask can be solved with more accuracy and less computation. For a real baseband OFDM system with $\mathrm{N}=2 \mathrm{M}$ ( $\mathrm{M}$ a positive integer) subcarriers and a

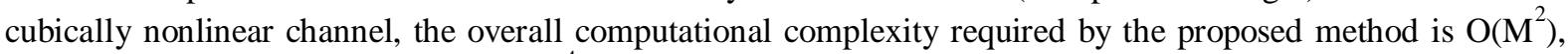
which is significantly less than the $\mathrm{O}\left(\mathrm{M}^{4}\right)$ for the conventional method described in [13], [14]. The goodness of the proposed method is also justified by computer simulations.

\section{Real Baseband Ofdm Systems}

A baseband OFDM signal is defined as:

$$
x(n)=\frac{1}{N} \sum_{m=0}^{N-1} X(m) e^{j 2 \pi n m / N}, n=0,1, \ldots, N-1
$$

where $\{\mathrm{X}(\mathrm{m})\}$ are the complex data symbols and $\mathrm{N}$ is the number of subcarriers. For a sampling period of Ts, the above OFDM signal has a waveform duration of $\mathrm{T}=\mathrm{N}$ Ts. The subcarriers are equally spaced and separated by $\Delta f=1 / T$. For wire line communications such as PLC and ADSL, the baseband OFDM signal is transmitted without further frequency up conversion. Therefore, the OFDM signal $\mathrm{x}(\mathrm{n})$ has to be real. To comply with this constraint, the symbols $\{X(m)\}$ need to be conjugate symmetric with respect to the discrete frequency N/2 [1]. In addition, $X(0)$ and $X(N / 2)$ need to be real. This implies that only N/2 - 1 independent complex data symbols can be transmitted in each frame of real OFDM signal with $\mathrm{N}$ subcarriers. The block diagram of a real OFDM system is shown in Fig. 1. In the transmitting end, the bit stream $b[n]$ is first converted to parallel bit streams (via the serial-to-parallel (S/P) converter) according to the available subcarriers in the OFDM system. Each bit stream is further mapped to the symbol stream according to the chosen modulation scheme (e.g., PSK or QAM). Here the $\mathrm{m}$-th symbol stream is denoted by $\mathrm{X}(\mathrm{m})$. The $\mathrm{N}$ parallel symbols for the $\mathrm{N}$ subcarriers are then processed by an inverse fast Fourier transform (IFFT) to obtain the time-domain baseband OFDM signal $x(n)$. The baseband signal is filtered, converted to an analog signal (via the $\mathrm{D} / \mathrm{A}$ ), boosted by the power amplifier (PA), and then sent via the wire line channel. The OFDM receiver performs analog-to-digital conversion (A/D), filtering, and fast Fourier transform (FFT) to obtain the output symbols $\{\mathrm{Y}(\mathrm{m})\}$ for the $\mathrm{N}$ sub channels. The output symbols $\{\mathrm{Y}(\mathrm{m})\}$ are further detected to obtain the optimum estimates of $\{\mathrm{X}(\mathrm{m})\}$, say, $\left\{\mathrm{X}^{\wedge}(\mathrm{m})\right\}$. Finally, the detected bit stream ${ }^{\wedge}[\mathrm{n}]$ can be obtained from $\left\{\mathrm{X}^{\wedge}(\mathrm{m})\right\}$ through bit mapping and parallel-to-serial (P/S) conversion.

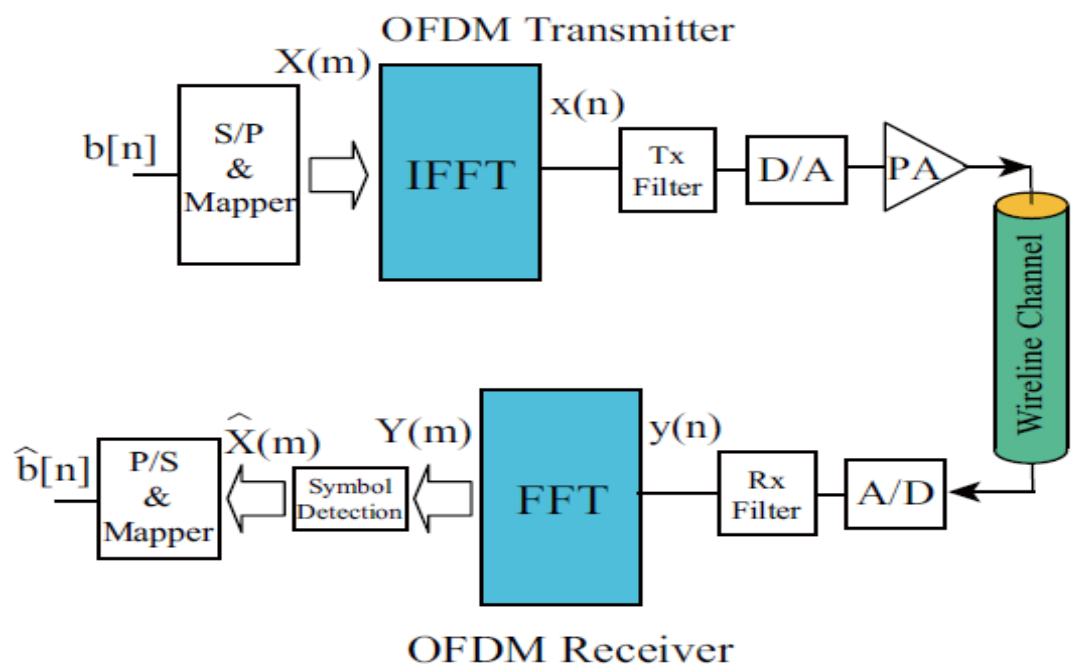

Figure. 1. The block diagram of a real OFDM system. 


\section{Mmse Estimate Of The Frequency-Domainvolterra Kernels}

Due to the existence of the PA in Fig. 1, the baseband OFDM communication channel may become nonlinear. Suppose that the subcarrier spam a bandwidth of W. Due to the possible bandwidth broadening characteristics of the nonlinear channel, the channel output may have a bandwidth as wide $3 \mathrm{~W}$ [13]. If the input and output signals were sampled from aliasing, then the discrete frequency-domain third-order Volterra series representation of the OFDM system can be written as follows [13]:

$$
\begin{array}{r}
Y(m)=Y_{1}(m)+Y_{2}(m)+Y_{3}(m)+\varepsilon(m), \quad-3 M \leq m \leq 3 M \\
Y_{1}(m)= \begin{cases}H_{1}(m) X(m), \quad|m| \leq M \\
0, \quad M+1 \leq|m| \leq 3 M\end{cases} \\
Y_{2}(m)=\left\{\begin{array}{cc}
\sum_{-M \leq i, j \leq M} H_{2}(i, j) X(i) X(j), \quad|m| \leq 2 M \\
(i+j=m) \\
0, \quad 2 M+1 \leq|m| \leq 3 M
\end{array}\right. \\
Y_{3}(m)=\sum_{-\begin{array}{c}
M \leq i, j, k \leq M \\
(i+j+k=m)
\end{array}} H_{3}(i, j, k) X(i) X(j) X(k), \quad|m| \leq 3 M
\end{array}
$$

where $X(m)$ and $Y(m)$ are the discrete Fourier transforms (DFTs) of the input and output, and $Y_{1}(m), Y_{2}(m)$, and $\mathrm{Y}_{3}(\mathrm{~m})$ are the linear, quadratic, and cubic responses of the system. The linear, quadratic, and cubic Volterra kernels of the systemare denoted by $\mathrm{H}_{1}(\mathrm{~m}), \mathrm{H}_{2}(\mathrm{i}, \mathrm{j})$, and $\mathrm{H}_{3}(\mathrm{i}, \mathrm{j}, \mathrm{k})$, respectively, in (3)-(5). The modeling error is denoted by $\varepsilon(\mathrm{m})$, and the maximum discrete frequency of $X(\mathrm{~m})$ with a non-zero value is denoted by $\mathrm{M}$.

However, in an OFDM system like that shown in Fig. 1, the sampling frequency $f_{s}$ is often set to $2 \mathrm{~W}$ instead of $6 \mathrm{~W}$. Under the circumstances, the resulting output data samples are aliased. According to the sampling theorem, those quadratic and cubic output terms that were contributing to frequencies outside the range of $m \in$ $[-\mathrm{M}, \mathrm{M}]$ in (4) and (5) will now wrapped around to show up in the range $\mathrm{m} \in[-\mathrm{M}, \mathrm{M}]$ as aliases. Therefore, the total quadratic and cubic outputs, say, $\mathrm{Yq}(\mathrm{m})$ and $\mathrm{Yc}(\mathrm{m})$, in $\mathrm{Y}(\mathrm{m})$ can be written as follows [14]:

$$
\begin{array}{cl}
Y_{q}(m)=Y_{2}(m)+Y_{2}(-N+m), & 0 \leq m \leq M \\
Y_{c}(m)=Y_{3}(m)+Y_{3}(m+N)+Y_{3}(-N+m), & 0 \leq m \leq M
\end{array}
$$

Where $\mathrm{Y}_{2}(-\mathrm{N}+\mathrm{m}), \mathrm{Y}_{3}(\mathrm{~m}+\mathrm{N})$, and $\mathrm{Y}_{3}(-\mathrm{N}+\mathrm{m})$ are the alias terms. In (6) and (7), only non-negative frequencies are considered because $\mathrm{Y}_{\mathrm{q}}(\mathrm{m})$ and $\mathrm{Y}_{\mathrm{c}}(\mathrm{m})$ are conjugate symmetric with respect to the frequency 0 .

The above analysis suggests that, when the sampling frequency is $2 \mathrm{~W}$, the relation between $\mathrm{X}(\mathrm{m})$ and $\mathrm{Y}(\mathrm{m})$ described by (2) is no longer appropriate and should be modified as follows [14]:

$$
Y(m)=Y_{1}(m)+Y_{q}(m)+Y_{c}(m)+\varepsilon(m),
$$

$$
0 \leq m \leq M
$$

If we arrange all the involved Volterra kernel coefficients in (8) into a vector $\mathrm{H}(\mathrm{m})$, and all their corresponding input terms into a vector $\mathrm{X}(\mathrm{m})$, we can rewrite (8) as

$$
Y(m)=X^{T}(m) H(m)+\varepsilon(m), 0 \leq m \leq M(9)
$$

By minimizing the mean square error $E\left[|\varepsilon(m)|^{2}\right]$ in (9), one can obtain the optimal minimum mean square error (MMSE) estimate of $\mathrm{H}(\mathrm{m})$, say $\mathrm{H}^{\wedge}(\mathrm{m})$, as follows:

$$
\widehat{H}(m)=E\left[X^{*}(m) X^{T}(m)\right]^{-1} E\left[X^{*}(m) Y(m)\right],
$$

$$
0 \leq m \leq M
$$

Note that $\mathrm{E}\left[\mathrm{X} *(\mathrm{~m}) \mathrm{X}^{\mathrm{T}}(\mathrm{m})\right]$ is composed of higher-order auto-moment spectra of $\mathrm{X}(\mathrm{m})$ up to 6th order, and $\mathrm{E}[\mathrm{X} * \mathrm{~m}) \mathrm{Y}(\mathrm{m})]$ is composed of higher-order cross-moment spectra between $\mathrm{X}(\mathrm{m})$ and $\mathrm{Y}(\mathrm{m})$ up to 4th order. The reader is referred to [14] for further details.

Note that calculation of an inverse matrix is required in (10). The computational complexity of inverting a $\mathrm{K}$ $\times \mathrm{K}$ matrix is $\mathrm{O}\left(\mathrm{K}^{3}\right) \quad$ [19]. By taking into account the symmetry properties of the Volterra kernel, we see that there are about $\mathrm{M}$ quadratic kernel coefficients involved in (6), and about $\mathrm{M}^{2}$ cubic kernel coefficients involved in (7). Since the cubic kernel coefficients dominate the vector $H(m)$, the size of the vector $H(m)$ (and hence $\mathrm{X}(\mathrm{m})$ ) is approximately $\mathrm{M}^{2}$. This suggests that the computation of the inverse matrix in (10) has an $\mathrm{O}\left(\mathrm{M}^{6}\right)$ complexity. We will refer to (10) as the general input method hereafter.

\section{The Spectral Notch Method}

In this section, we present a novel method to estimate the frequency-domain Volterra kernels of nonlinear channels in OFDM systems using OFDM signals with spectral notches. In this method, each OFDM symbol frame with a full spectrum is followed by $\mathrm{M}+1$ copies of the same OFDM symbol frame, but the $\mathrm{p}$-th copy $(p=0,1, \ldots, M)$ has a missing spectral component at the $p$-th subcarrier. Here we imply that the spectral component at the (N-p)-th subcarrier is also missing in the $\mathrm{p}$-th copy. This is due to the fact that the OFDM 
signal is real and it's spectrum must be conjugate symmetric with respect to the frequency N/2. An OFDM symbol frame with a full spectrum is shown in Fig. 2(a) (denoted by X(m)). The same OFDM symbol frame with the spectral line at the p-th subcarrier missing is shown in Fig. 2(b) (denoted by $\mathrm{X}^{(\mathrm{p})}(\mathrm{m})$ ). As we can see in Fig. 2(b), the missing spectral line yields a spectral notch at the discrete frequency $\mathrm{p}$ in the spectrum of the OFDM signal. Therefore, the method presented here is referred to as the spectral notch method.

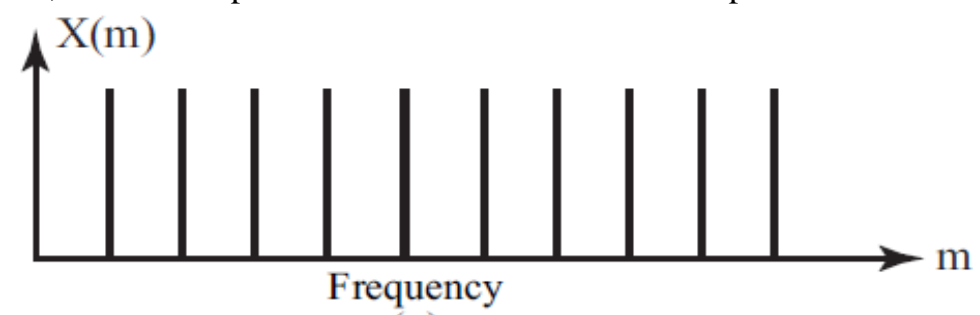

(a)

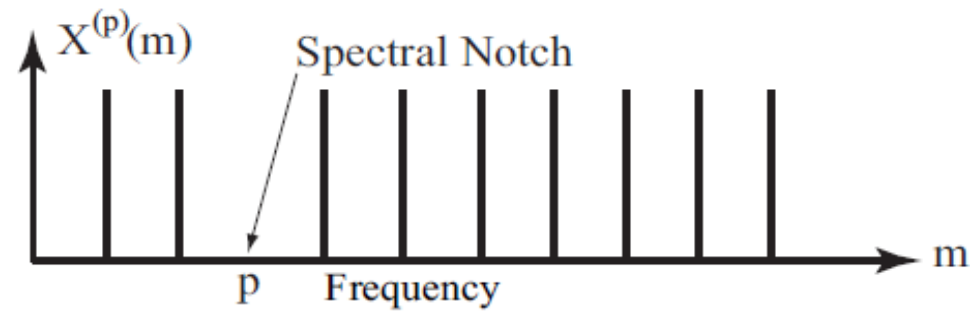

(b)

Figure. 2. (a) The OFDM symbol frame with a full spectral and (b) the same OFDM symbol frame with the spectral line at the p-th subcarrier missing

Passing the OFDM symbol frame $\mathrm{X}(\mathrm{m})$ in Fig. 2(a) through the nonlinear channel in Fig. 1 may yield an output $\mathrm{Y}(\mathrm{m})$ like that shown in Fig. 3(a), where $\mathrm{Y}(\mathrm{m})$ and $\mathrm{X}(\mathrm{m})$ are related by (8). Similarly, passing the OFDM symbol frame $\mathrm{X}^{(\mathrm{p})}(\mathrm{m})$ in Fig. 2(b) through the nonlinear channel in Fig. 1 may yield an output $\mathrm{Y}^{(\mathrm{p})}(\mathrm{m})$ like that shown in Fig. 3(b). Note that, due to the spectral notch at frequency p in Fig. 2(b), the spectral line appears at frequency p in Fig. 3(b) is completely owing to nonlinear distortions (and perhaps plus noise) of the channel. Since $\mathrm{X}^{(\mathrm{p})}(\mathrm{m})$ and $\mathrm{X}(\mathrm{m})$ are basically the same except at frequency $\mathrm{p}$, the relation between $\mathrm{Y}^{(\mathrm{p})}(\mathrm{m})$ and $\mathrm{X}(\mathrm{m})$ can be obtained by modifying $(8)$ as follows:

Where

$$
\begin{gathered}
Y^{(P)}(m)=Y_{1}^{(P)}(m)+Y_{2}^{(p)}(m)+Y_{2}^{(p)}(-N+m)+Y_{3}^{(p)}(m)+Y_{3}^{(p)}(m+N)+Y_{3}^{(p)}(-N+m) \\
+\varepsilon^{(p)}(m)
\end{gathered}
$$

$$
\begin{gathered}
Y_{1}^{(p)}(m)=\left\{\begin{array}{cc}
H_{1}(m) X(m),|m| \leq M,|m| \neq p \\
0, \quad M+1 \leq|m| \leq 3 M
\end{array}\right. \\
Y_{2}^{(p)}(m)=\left\{\begin{array}{c}
\sum_{\substack{-M \leq i, j \leq M \\
(i+j=m,|i|,|j| \neq p)}} H_{2}(i, j) X(i) X(j), \quad|m| \leq 2 M \\
0, \quad 2 M+1 \leq|m| \leq 3 M
\end{array}\right. \\
Y_{3}^{(p)}(m)=\sum_{\substack{-M \leq i, j, k \leq M \\
(i+j+k=m,|i|,|j|,|k| \neq p) \\
|m| \leq 3 M}} H_{3}(i, j, k) X(i) X(j) X(k),
\end{gathered}
$$



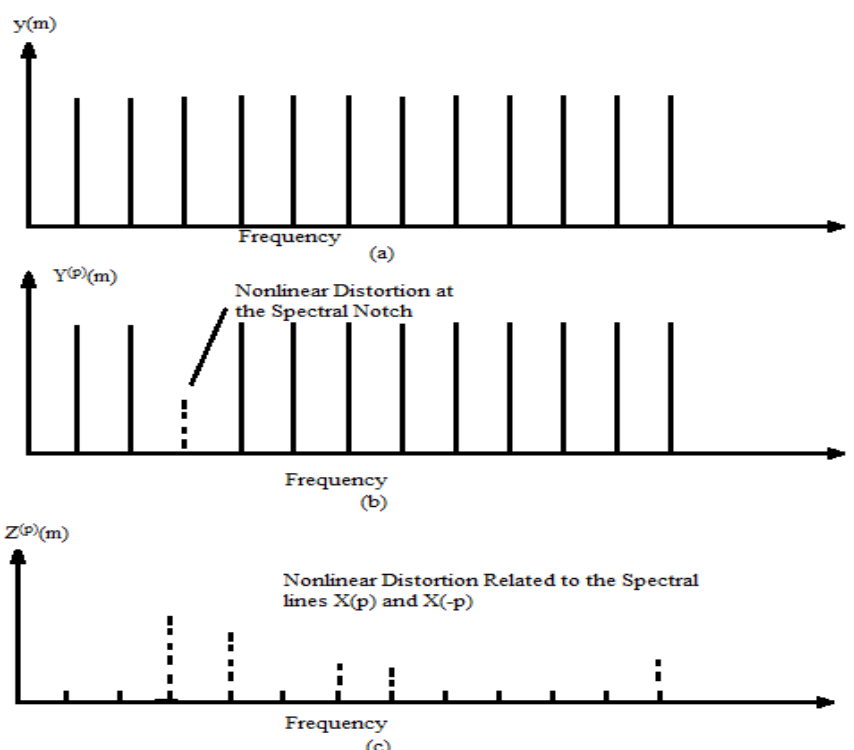

Figure. 3. (a) The output spectrum $Y(m)$ for theOFDM symbol frame $X(m)$.

(b) The output spectrum $\mathrm{Y}^{(\mathrm{p})}(\mathrm{m})$ for theOFDM symbol frame $\mathrm{X}^{(\mathrm{p})}(\mathrm{m})$

(c) The difference spectrum $\mathrm{Z}^{(\mathrm{p})}(\mathrm{m})$ between $\mathrm{Y}(\mathrm{p})$ and $\mathrm{Y}^{(\mathrm{p})}(\mathrm{m})$

Basically, $Y_{1}^{(p)}(m), Y_{2}^{(p)}(m)$ and $Y_{3}^{(p)}(m)$ are obtained from $Y_{1}(m), Y_{2}(m)$, and $Y_{3}(m)$, respectively, by eliminating those terms involving $X(p)$ and $X(-p)$ in (3)-(5). One can compare (12)-(14) to (3)-(5) to see the differences. Next we subtract $Y^{(\mathrm{p})}(\mathrm{m})$ from $Y(\mathrm{~m})$ to obtain their difference spectrum $\left.\mathrm{Z}^{(\mathrm{p})} \mathrm{m}\right)$. The result would be like that shown in Fig. 3(c). Note that all the spectral lines in Fig. 3(c) are completely owing to nonlinear distortions related to the spectral lines $\quad X(p)$ and $X(-p)$ in Fig. 2(a). The difference spectrum $Z^{(p)}(m)$ can be mathematically expressed as:

Where

$$
\begin{gathered}
Z^{(p)}(m)=Z_{1}^{(p)}(m)+Z_{2}^{(p)}(m)+Z_{2}^{(p)}(-N+m)+Z_{3}^{(p)}(m)+Z_{3}^{(p)}(m+N)+Z_{3}^{(p)}(-N+m) \\
+\varphi^{(p)}(m)
\end{gathered}
$$

$$
\begin{aligned}
& Z_{1}^{(p)}(m)=\left\{\begin{array}{l}
H_{1}(m) X(m), \quad|m|=p \\
0, \quad \text { otherwise }
\end{array}\right.
\end{aligned}
$$

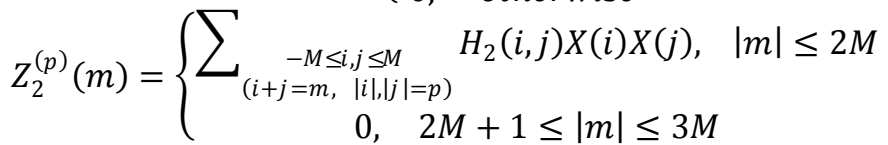

$$
\begin{aligned}
& Z_{3}^{(p)}(m)=\sum_{-M \leq i, j, k \leq M} H_{3}(i, j, k) X(i) X(j) X(k), \quad|m| \leq 3 M
\end{aligned}
$$

Note that the right-hand sides of (16)-(18) in fact contain only those terms involving $\mathrm{X}(\mathrm{p})$ or $\mathrm{X}(-\mathrm{p})$ in (3)-(5), respectively. The error term $\psi^{(p)}(m)$ in $(15)$ is defined as $\psi^{(p)}(m)=\varepsilon(m)-\varepsilon^{(p)}(m)$

By arranging all the Volterra kernel coefficients in (15) into a vector $H^{(p)}(m)$, and all their corresponding input terms into a vector $X^{(p)}(m)$ we can rewrite (15) as

$$
Z^{(p)}(m)=X^{(p)^{T}}(m) H^{(p)}(m)+\psi^{(p)}(m)
$$

By minimizing the mean square error $\mathrm{E}\left[\psi^{(p)}(m)\right]^{2}$, one can obtain the optimum MMSE estimate of $\mathrm{H}^{(\mathrm{p})}(\mathrm{m})$ as follows:

$$
\widehat{H}^{(p)}(m)=E\left[X^{(p) *}(m) X^{(p)^{T}}(m)\right]^{-1} E\left[X^{(p) *}(m) Z^{(p)}(m)\right], 0 \leq m \leq M, 0 \leq p \leq M
$$

After taking into account the symmetry properties of the Volterra kernels, we see that there are at most 2 distinct quadratic kernel coefficients and $\mathrm{M}$ distinct cubic kernel coefficients involved in (17) and (18), respectively. Since the cubic kernel coefficients dominate the vector $\mathrm{H}^{(\mathrm{p})}(\mathrm{m})$, the size of the vector $\mathrm{H}^{(\mathrm{p})}(\mathrm{m})$ (and hence $\left.X^{(p)}(m)\right)$ is approximately $M$. This suggests that the complexity of inverting the matrix in (20) is $O\left(M^{3}\right)$. Note that $\mathrm{H}^{(\mathrm{p})}(\mathrm{m})$ only includes those kernel coefficients contributing to $\mathrm{Y}(\mathrm{m})$ and excited by $\mathrm{X}(\mathrm{p})$ or $\mathrm{X}(-\mathrm{p})$. By varying $\mathrm{p}$ from 0 to $\mathrm{M}$, we can cover all the kernel coefficients contributing to $\mathrm{Y}(\mathrm{m})$. This implies that, to get estimates for all the kernel coefficients contributing to $\mathrm{Y}(\mathrm{m})$, the computational complexity required by the spectral notch method is $\mathrm{M} \times \mathrm{O}\left(\mathrm{M}^{3}\right)=\mathrm{O}\left(\mathrm{M}^{4}\right)$. This is a significant saving compared to the $\mathrm{O}\left(\mathrm{M}^{6}\right)$ required by 
the general input method. Furthermore, as we vary $\mathrm{p}$ from 0 to $\mathrm{M}$, some of the kernel coefficients included in $\mathrm{H}^{(\mathrm{p})}(\mathrm{m})$ may have already appeared in $\mathrm{H}^{(\mathrm{r})}(\mathrm{m})(\mathrm{r} \in[0, \mathrm{p}))$ and been estimated earlier. These kernel coefficients don't need to be estimated again in $\mathrm{H}^{(\mathrm{p})}(\mathrm{m})$. For example, the cubic kernel coefficient $\mathrm{H}(0,1,2)$ is excited by $X(0), X(1)$, and $X(2)$, therefore, it would show up in ${ }^{(0)}(3), H^{(1)}(3)$, and $H^{(2)}(3)$. Since this coefficient would have been estimated in $\mathrm{H}^{(0)}(3)$, it doesn't need to be estimated again when estimating $\mathrm{H}^{(1)}(3)$ and $\mathrm{H}^{(2)}(3)$. Therefore, for those kernel coefficients in $\mathrm{H}^{(\mathrm{p})}(\mathrm{m})$ that have already been estimated earlier, we can substitute their estimated values into (19) and eliminate them from $\mathrm{H}^{(\mathrm{p})}(\mathrm{m})$. As a result, the size of the vector $\mathrm{H}^{(\mathrm{p})}(\mathrm{m})$ is reduced. That is, the size of $\mathrm{H}^{(\mathrm{p})}(\mathrm{m})$ will keep decreasing as we vary $\mathrm{p}$ from 0 to $\mathrm{M}$. This further reduces the computational complexity of the spectral notch method.

\section{Simulation Results}

To demonstrate the goodness of the spectral notch method, we conducted simulations using the following cubically non-linear system [14]:

$$
y(n)=-0.64 x(n)+x(n-2)+0.9 x^{2}(n)+x^{2}(n-1)+0.6 x^{3}(n)-0.3 x^{3}(n-1)+e(n)
$$

The frequency-domain Volterra kernels of this system are given by

$$
\begin{gathered}
H_{1}(m)=-0.64+e^{-j 4 \pi m / N}(22) \\
H_{2}\left(i_{1}, i_{2}\right)=0.9+e^{-j 2 \pi\left(i_{1}+i_{2}\right) / N}(23) \\
H_{3}\left(i_{1}, i_{2}, i_{3}\right)=0.6-0.3 e^{-j 2 \pi\left(i_{1}+i_{2}+i_{3}\right) / N}(24)
\end{gathered}
$$

The input $\mathrm{x}(\mathrm{n})$ to the nonlinear system was an OFDM signal generated using (1) with $\mathrm{N}=32$. The complex symbols used in subcarriers 1 to 15 were 16 QAM. To ensure $\mathrm{x}(\mathrm{n})$ to be a real signal, the complex symbols for subcarriers 17 to 31 were the conjugate symmetric copies of those in subcarriers 1 to 15 , and the symbols for subcarriers 0 and 16 were real random numbers. The additive noise e(n) was a zero-mean, white, and Gaussian random signal. The signal to noise ratios (SNRs) were set to 0,10 , and $20 \mathrm{~dB}$.

For the input signal used in the general input method, every OFDM symbol frame had a full spectrum. A total of 18000 frames of the input and output data were collected and used by the general input method. The normalized mean square errors (NMSEs) of the estimated Volterra kernels (including linear, quadratic, and cubic kernels) achieved by the general input method (GEN) using 900, 1800, 9000, and 18000 frames of data and under the SNRs of 0,10 , and $20 \mathrm{~dB}$ are shown in Fig. 4 (denoted by $\times$ ). The NMSE of the Volterra kernel estimate was defined as:

$$
N M S E=\frac{\|c-h\|^{2}}{\|c\|^{2}}
$$

Where $\mathrm{c}$ and $\mathrm{h}$ are vectors including all the actual and estimated Volterra kernel coefficients, respectively, and $\|\cdot\|$ is the norm operator.

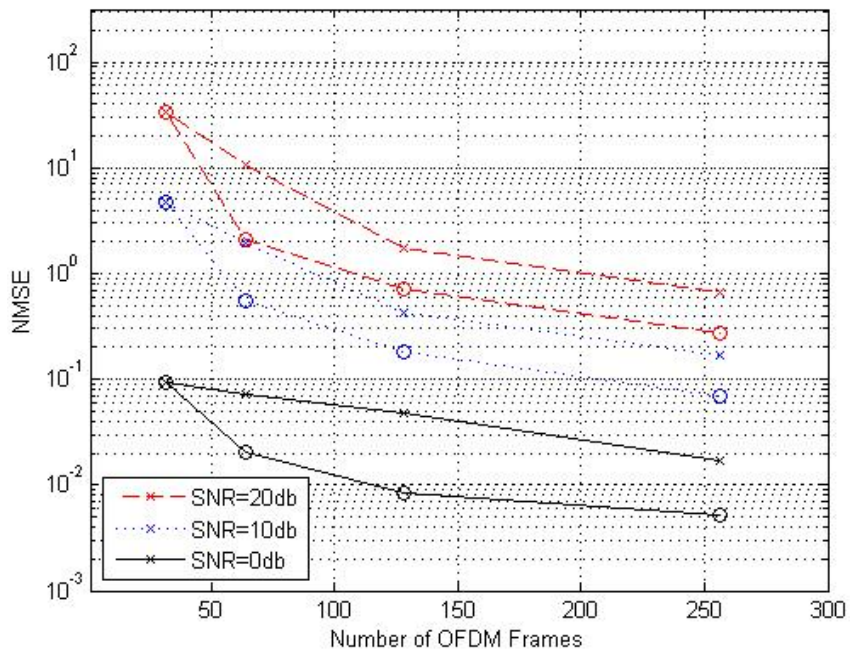

Figure. 4. The NMSEs of the Volterra kernel estimates achieved by the generalinput method (GEN) and the spectral notch method (NOT) under the SNRsof 0, 10, and $20 \mathrm{~dB}$ using various number of OFDM symbol frames.

For the input signal used in the spectral notch method, every OFDM symbol frame with a full spectrum was followed by 17 copies of the same OFDM symbol frame, but each copy had a distinct spectral notch at the frequency $\mathrm{p} \in[0,16]$. The system output of each copy was subtracted from that of the corresponding full-spectral OFDM symbol frame to obtain the $Z^{(\mathrm{p})}(\mathrm{m})$ in $(15)$. We conducted cases with 50, 100, 
500, and 1000 full-spectral OFDM symbol frames, which leaded to total OFDM symbol frames (including those with spectral notches) of 900, 1800, 9000, and 18000, respectively. The NMSEs of the estimated Volterra kernels achieved by the spectral notch method (NOT) for the 4 cases under the SNRs of 0, 10, and $20 \mathrm{~dB}$ are also shown in Fig. 4 (denoted by $\circ$ ).

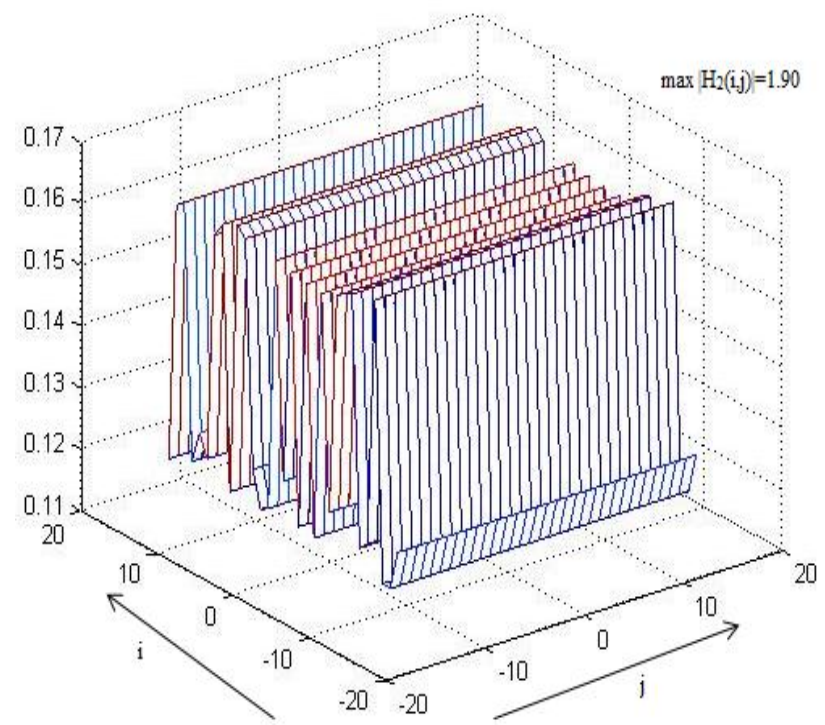

Figure. 5. The amplitude response of the actual quadratic Volterra kernel.

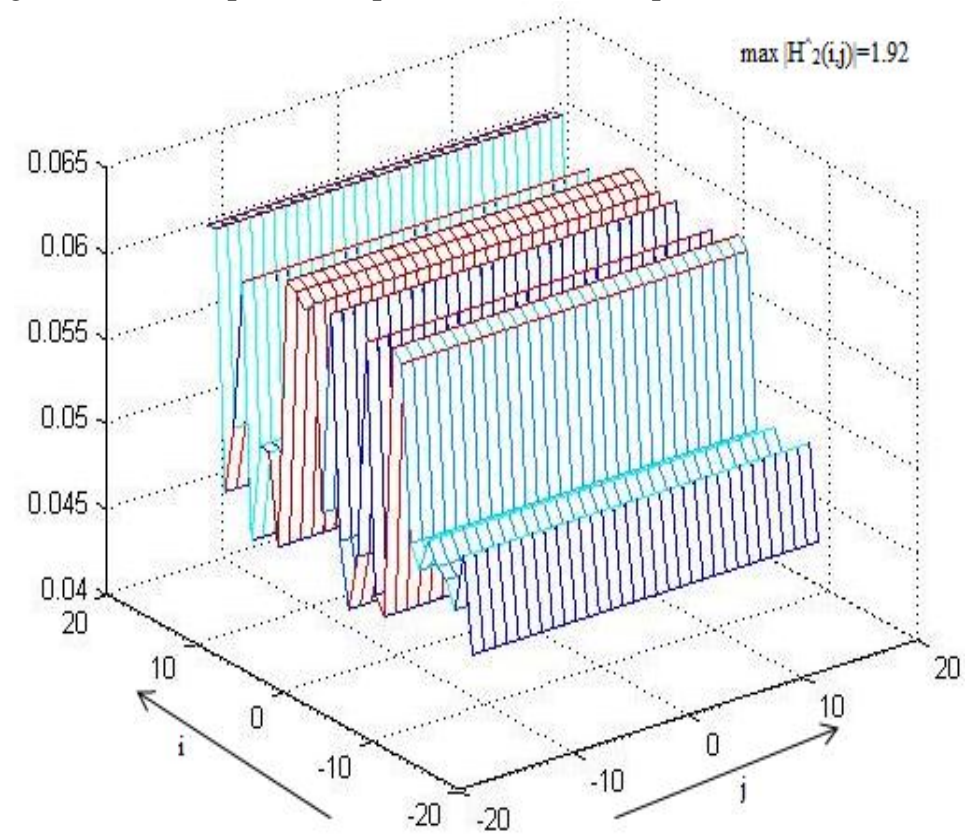

Figure. 6. The amplitude response of the estimated quadratic Volterra kernal

From Fig. 4 we see that, under the same SNR and number of data frames, the spectral notch method achieved a smaller NMSE than the general input method. One possible reason for this is that, in the general input method, all the Volterra kernel coefficients contributing to the output at a frequency $\mathrm{m}$ are jointly estimated. On the other hand, through the use of spectral notches, the Volterra kernel coefficients contributing to the output at the frequency $\mathrm{m}$ are decomposed into disjoint groups in the spectral notch method. Compared to the general input method, each group in the spectral notch method only involves a relatively small number of Volterra kernel coefficients, hence the spectral notch method can estimate the Volterra kernels more accurately given the same amount of data. The result shown in Fig. 4 also seems to suggest that the performance discrepancy between the spectral notch and general input methods would increase with the number of available OFDM symbol frames.

The NMSEs of the estimated total, linear, quadratic, and cubic Volterra kernels achieved by the spectral notch method using 18000 frames of data and under SNR=20 dB are $4.97 \times 10^{-4}, 3.48 \times 10^{-2}, 9.91 \times 10^{-5}$, and $7.43 \times 10^{-5}$, respectively. The actual and the estimated quadratic Volterra kernels in the estimation region are 
shown in Figs. 5 and 6, respectively.

We also conducted a simulation with 256 subcarriers for the same system described by (21). In this case, the general input method couldn't even be executed on our computer due to its extremely high demand on memory. The spectral notch method, on the other hand, achieved a NMSE of $1.20 \times 10^{-3}$ under SNR=20 dB and using 65000 frames of data.

\section{Conclusion}

In this paper, we have proposed a practical methodology to estimate nonlinear channels in OFDM systems by deploying spectral notches in the OFDM signal. By exploring the mathematical expression for the difference signal between the outputs of a full-spectral OFDM symbol frame and its corresponding OFDM symbol frame with a spectral notch, we have shown that the Volterra kernel coefficients contributing to the output at any given frequency can be decomposed into disjoint groups with smaller numbers of kernel coefficients. As a result,Volterra kernel coefficients belonging to different groups can be estimated independently. Since each group involves only a relatively small number of Volterra kernel coefficients, the Volterra kernels can be estimated more accurately by the proposed method using the same amount of available input and output data. Furthermore, due to the smaller number of Volterra kernel coefficients involved in each group, the proposed spectral notch method requires significantly less computation than the conventional general input method. Specifically, we have shown that, the conventional general input method has a computational complexity of $\left.\mathrm{O} \mathrm{M}^{6}\right)$, while the proposed spectral notch method only requires a computational complexity of $\mathrm{O}\left(\mathrm{M}^{4}\right)$. The advantage of the proposed spectral notch method over the conventional general input method has been justified by the computer simulations.

\section{References}

[1] A. R. S. Bahai, B. R. Saltzberg, and M. Ergen, Multi-Carrier DigitalCommunications Theory and Applications of OFDM, 2nd ed., New York,NY: Springer, 2004

[2] HomePlugPowerline Alliance, Medium Interface Specification Release0.5, Nov. 2000.

[3] ITU-T, "Asymmetric digital subscriber line (ADSL) transceivers," ITU-TRecommendation G.992.1, Geneva, 1999.

[4] IEEE, "Wireless LAM medium access control (MAC) and physical Layer(PHY) specifications: High-speed physical layer in the 5 GHz band,"IEEE Standard 802.11a-1999, Sep. 1999.

[5] ETSI, "Digital audio broadcasting (DAB), guidelines and rules forimplementation and operation, part 1: system outline," ETSI Technical Report, TR 101 496-1 v1.1.1, Nov. 2000.

[6] ETSI, "Digital video broadcasting (DVB), framing structure, Channel coding and modulation for digital terrestrial television,"ETSIEuropean Standard, EN 300744 v1.2.1, Jul. 1999.

[7] M. Schetzen, The Volterra and Wiener Theories of Nonlinear System.New York: Wiley, 1980.

[8] W. J. Rugh, Nonlinear System Theory - The Volterra/Wiener Approach,London: The Johns Hopkins Press Ltd., 1981.

[9] A. S. French and E. G. Butz, "Measuring the Wiener kernels of a non linear system using the fast Fourier transform algorithm," Int. J. Contr.,vol. 17, no. 3, pp. 529-539, 1973

[10] L. J. Tick, “The estimation of transfer functions of quadratic systems," Technometrics, vol. 3, pp. 563-567, Nov. 1961.

[11] J. S. Bendat, Nonlinear System Analysis and Identification From Random Data, New York: Wiley, 1990.

[12] V. J. Mathews and G. L. Sicuranza, Polynomial Signal Processing, New York: John Wiley \& Sons, 2000.

[13] S. W. Nam and E. J. Powers, "Application of higher order spectralanalysis to cubically nonlinear system identification," IEEE Trans. Signal Processing, vol. 42, no. 7, July 1994.

[14] C.-H. Tseng, "Identification of cubically nonlinear systems using under-sampled data,"IEE Proc.-Vis. Image Signal Process., vol. 144, no. 5,pp. 267-277, Oct. 1997.

[15] C. L. Nikias and M. R. Raghuveer, "Bispectrum estimation: a digitalsignal processing framework," Proc. IEEE, vol. 75, no. 7, pp. 869-891,July 1987.

[16] D. R. Brillinger, "Identification of polynomial systems by means ofhigher-order spectra," J. Sound, Vibration, vol. 12, pp. 301-313, 1970.

[17] A. J. Redfern and G. T. Zhou, "Nonlinear channel identification andequalization for OFDM systems," Proc. IEEE Int. Conf. Acoust. Speech,Signal Process., vol. 6, pp. 3521-3524, May 12-15, 1998.

[18] G. Mileounis, P. Koukoulas, and N. Kalouptsidis, "Input-outputidentification of nonlinear channels using PSK, QAM, and OFDMinputs,"Signal Process., vol. 89, no. 7, pp. 1359-1369, July 2009.

[19] G. H. Golub and C. F. Van Loan, Matrix Computations, 2nd ed.,The Baltimore, MD: Johns Hopkins University Press, 1989. 